OUTP-98-09P

June 1998

\title{
The effect of Yukawa couplings on Unification Predictions and the non-perturbative limit
}

\author{
G. Amelino-Camelia*,t, \\ * Department of Physics, Theoretical Physics, University of Oxford \\ 1 Keble Road, Oxford OX1 3NP, United Kingdom \\ † Institut de Physique, Université de Neuchâtel, \\ rue Breguet 1, CH-2000 Neuchâtel, Switzerland
}

\begin{abstract}
We investigate the effects of Yukawa couplings on the phenomenological predictions for a class of supersymmetric models which allows for the presence of complete $S U(5)$ multiplets in addition to the Minimal Supersymmetric Standard Model spectrum. We develop a two-loop analytical approach to quantify the predictions for gauge unification including Yukawa couplings. The effects of the heavy thresholds of the model are also included. In some cases accurate predictions can be made for the unification scale, irrespective of the initial (unknown) Yukawa couplings, so long as perturbation theory remains valid. We also consider the limit of a large number of extra states and compute the predictions in a resummed perturbation series approach to show that the results are stable in this limit. Finally we consider the possibility of making predictions for the case the gauge and Yukawa couplings enter the non-perturbative domain below the unification scale and estimate the errors which affect these predictions.
\end{abstract}

\footnotetext{
${ }^{1}$ E-mail address: Giovanni.Amelino-Camelia@cern.ch

${ }^{2}$ E-mail address: D.Ghilencea1@physics.oxford.ac.uk

${ }^{3}$ E-mail address: G.Ross1@physics.oxford.ac.uk
} 


\section{Introduction}

In a recent paper [1] the phenomenological implications of a class of string motivated supersymmetric models based on level-1 heterotic string models with Wilson line breaking of the underlying $\mathrm{E}_{6}$ symmetry were investigated. These models (henceforth referred to as Extended Minimal Supersymmetric Models (EMSSM)) contain additional vector-like representations filling out complete five and ten dimensional representations of $S U(5)$ even though the gauge group is just that of the Standard Model]. This class of string models has several advantages for model building [2]; the Wilson line breaking necessary to break the $E_{6}$ symmetry in level-1 theories offers an elegant way out of the doublet-triplet splitting problem encountered in GUTs. It can also lead to the standard unification of the gauge couplings without the need of a Grand Unified Group below the compactification scale 2 . In this case the string prediction for the scale of unification of the gauge couplings may be directly compared with the value obtained continuing the gauge couplings up in energy providing the possibility of a quantitative test of string unification including gravity.

The effect of the additional vector-like states on $\alpha_{3}\left(M_{z}\right)$ and the value of the unification scale was explored in detail in [1]. It was found that, working to two-loop order in the gauge sector, the unification scale is systematically increased by a small factor (about 3 or less, a function of the value of the unified coupling and of the number $n=\left(n_{5}+3 n_{10}\right) / 2$ of pairs of additional complete $S U(5)$ representations), while the strong coupling was systematically increased, taking it further from the experimental value 顿, $\alpha_{3}\left(M_{z}\right)=0.118 \pm 0.003$. The increase in the unification scale does not, in fact, take it closer to the weakly coupled heterotic string prediction [5] because the increase is correlated with an increase in the unified coupling which always happens when additional matter is added and the heterotic string prediction also increases with the unified coupling. As a result there is still a discrepancyf of a factor of 10-20. However, in this analysis the effects of Yukawa couplings on the running of the gauge couplings were not included and we seek to include them in this paper. An immediate difficulty arises because in theories with additional massive vectorlike states there may be many new Yukawa couplings involving these heavy states. We shall consider two basic possibilities which give an indication of the general possibilities and uncertainties. In the first we include only the Yukawa couplings present in the MSSM which are responsible for the third generation masses. In the second we include couplings between massive Higgs doublets and the light quarks and leptons. This model goes some way towards explaining the light quark mass matrix via mixing in the Higgs sector [7] as well as explaining the masses of the third generation, in a "fixed-point-scenario" [8]. We also comment on how our results would be affected if further couplings involving massive states were included.

The paper is organized as follows. In Section 2 we review the effect of heavy thresholds on the running coupling constants and we analyze the renormalisation group evolution (RGE) predictions for the gauge couplings with Yukawa couplings effects included. In section 3 we derive an analytic form for $\alpha_{3}\left(M_{z}\right)$ and the unification scale as well as the decoupling scale of the extra matter and make some numerical estimates. In section 4 we consider the case of large $n$. Finally, in Section 5 we consider the case of unification at strong coupling. We discuss the differences between the (quasi)-fixed point approach [8] and the two loop perturbative approach results. Our conclusions are presented in the last section.

\footnotetext{
${ }^{1}$ To be precise these models contain additional vector-like states $I+\bar{I}$, where $I, \bar{I}$ denote complete representations of $S U(5)$. The components $\psi$ of the additional complete $S U(5)$ representations transform under $S U(3)$ and $S U(2)$ groups as follows: for $\psi=d^{c}:(\overline{3}, 1), \psi=l:(1,2)$ for the case $I$ is the 5 dimensional representation of $S U(5)$ and $\psi=e^{c}$ : $(1,1), \psi=u^{c}:(\overline{3}, 1), \psi=q:(3,2)$ for the case $I$ is the 10 dimensional representation of $S U(5)$

${ }^{2}$ Alternative constructions were developed which can lead to non-standard U(1) normalisation even in level-1 theory see discussion in ref. 3]

${ }^{3} n_{5}=N_{5}+N_{\overline{5}}$ and $n_{10}=N_{10}+N_{\overline{10}}$

${ }^{4}$ Ways to reconcile this scale discrepancy have been reviewed by Dienes 6 .
} 


\section{Heavy thresholds' contribution from the integrated NSVZ beta function}

In this section we make use of the Novikov-Shifman-Vainshtein-Zakharov (NSVZ) beta function [9, 10] for the gauge couplings, which was derived using holomorphicity arguments and the instanton calculus. We integrate it in the presence of the additional heavy states of the EMSSM model and then use its two-loop approximation to perform further calculations.

The integrated expression for the gauge couplings' running, in the absence of these heavy thresholds due to the presence of the extra heavy states we consider, was presented in ref. [11] and was deduced without actually integrating the beta function, but by using physical arguments and the properties of the holomorphic gauge coupling; from that, the integrated form in the presence of our additional heavy thresholds can be easily "guessed". For clarity, we prefer to re-derive it here by direct integration. For a fuller discussion the reader is referred to the literature [10, 11, 12]. We have [9]

$$
\beta(\alpha)^{N S V Z} \equiv \frac{d \alpha}{d(\ln \mu)}=-\frac{\alpha^{2}}{2 \pi}\left[3 T(G)-\sum_{\sigma} T\left(R_{\sigma}\right)\left(1-\gamma_{\sigma}^{N S V Z}\right)\right]\left(1-T(G) \frac{\alpha}{2 \pi}\right)^{-1}
$$

with the definition ( $\mu$ is the running scale)

$$
\gamma_{\sigma}^{N S V Z}=-\frac{d \ln Z_{\sigma}}{d \ln \mu}
$$

and where $T(G)$ and $T\left(R_{\sigma}\right)$ represent the Dynkin index for the adjoint representation and for $R_{\sigma}$ representation respectively (not necessarily the fundamental one), whose values are given in the Appendix. The above sum runs over all matter fields $\sigma$ in representation $R_{\sigma}$ and this includes, for example, the extra heavy states (which we called $\psi$-see footnote (1)), in addition to the low energy spectrum of MSSM. This gives

$$
2 \pi \frac{d \alpha^{-1}}{d \ln \mu}+T(G) \frac{d \ln \alpha}{d \ln \mu}=3 T(G)-\sum_{\sigma} T\left(R_{\sigma}\right)\left(1-\gamma_{\sigma}^{N S V Z}\right)
$$

which can be integrated from the high scale $M$ down to the low scale $\mu$ with $\mu>\Lambda_{\text {Susy }}$ to give

$$
2 \pi\left(\alpha^{-1}(M)-\alpha^{-1}(\mu)\right)+T(G) \ln \frac{\alpha(M)}{\alpha(\mu)}=3 T(G) \ln \frac{M}{\mu}-\sum_{\sigma} \int_{\mu}^{M} T\left(R_{\sigma}\right)\left(1-\gamma_{\sigma}^{N S V Z}\right) d(\ln \tilde{\mu})
$$

In the above equation $\alpha$ stands for any gauge group coupling. Any extra heavy state $\psi$, with mass $\mu_{\psi}\left(\mu_{\psi}\right)$ larger than $\mu$, decouples at its physical mass scale in the running of the canonical gauge coupling [10, 12] and therefore, below $\mu_{\psi}$ scale, $\gamma_{\psi}=0$. From eq.(3) we obtain the result

$$
\begin{aligned}
\alpha^{-1}(\mu)=\alpha^{-1}(M) & +\frac{-3 T(G)}{2 \pi} \ln \frac{M}{\mu\left(\frac{\alpha(M)}{\alpha(\mu)}\right)^{1 / 3}} \\
& +\frac{1}{2 \pi} \sum_{\phi} T\left(R_{\phi}\right) \ln \frac{M}{\mu}+\frac{1}{2 \pi} \sum_{\phi} T\left(R_{\phi}\right) \ln \frac{Z_{\phi}(M)}{Z_{\phi}(\mu)} \\
& +\frac{1}{2 \pi} \sum_{\psi} T\left(R_{\psi}\right) \ln \frac{M}{\mu_{\psi}}+\frac{1}{2 \pi} \sum_{\psi} T\left(R_{\psi}\right) \ln \frac{Z_{\psi}(M)}{Z_{\psi}\left(\mu_{\psi}\right)}
\end{aligned}
$$

where $\phi$ 's stand for a MSSM-like spectrum. At this point we make the observation that

$$
\mu_{\psi} Z_{\psi}\left(\mu_{\psi}\right)=\mu_{\psi}^{o}
$$


which is the mass renormalisation equation, where $\mu_{\psi}^{o}$ represents the bare mass of the $S U(5)$ component $\psi$. For the case of a Grand Unified Group Model, the gauge invariance principle requires the mass terms $\mu_{\psi} \psi \bar{\psi}$ be invariant, which is possible only if all bare masses are equal to a common value, $\mu_{g}$. We also consider, without any loss of generality that $Z_{\psi}\left(M_{g}\right)=Z_{\phi}\left(M_{g}\right)=1$ where $M_{g}$ is the value of unification scale. Taking $M=M_{g}$, we find

$$
\begin{aligned}
\alpha^{-1}(\mu)= & \alpha^{-1}\left(M_{g}\right)+\frac{-3 T(G)}{2 \pi} \ln \frac{M_{g}}{\mu\left(\frac{\alpha\left(M_{g}\right)}{\alpha(\mu)}\right)^{1 / 3}}+\sum_{\phi} \frac{T\left(R_{\phi}\right)}{2 \pi} \ln \frac{M_{g}}{\mu Z_{\phi}(\mu)}+\frac{n}{2 \pi} \ln \frac{M_{g}}{\mu_{g}} \\
& +\frac{T\left(R_{\Sigma}\right)}{2 \pi} \ln \frac{M_{g}}{\mu_{\Sigma}}
\end{aligned}
$$

with $n=\left(n_{5}+3 n_{10}\right) / 2, n_{5}=N_{5}+N_{\overline{5}}$ and $n_{10}=N_{10}+N_{\overline{10}}$. The last term in eq.(5) could stand for the Higgs in the adjoint representation or for the $S U(3)$ Higgs triplet . This formula is valid to all orders in perturbation theory, as long as Supersymmetry is not broken. This equation was used, in this form, as a check of the intermediate results obtained in [1].

In two-loop order for gauge couplings running, $Z$ factors depend on the mean mass of the extra heavy states which can be considered to be their common bare mass $\mu_{g}$ since the difference would be of three loop order. Hence, in two-loop order $\mu_{g}$ is the only mass scale associated with the heavy states in eq.(5). Therefore one sees that the decoupling of the heavy states happens, in two-loop order, at $\mu_{g}$ and not at each of the physical masses of the component fields of the massive $S U(5)$ representations.

\section{Analytical Results from Renormalisation Group Evolution}

In this section we consider the effects of the Yukawa couplings on the running of the gauge couplings and their effects on the predictions for the unification scale, the strong coupling at electroweak scale and the scale where the heavy states decouple. In the case the Yukawa couplings are negligible, we recover previous two-loop analytical results [1].

To keep our approach general and to exhibit the effects Yukawa couplings have on the running of the gauge couplings, it is convenient to use eq.(5) for the running of the gauge couplings. We should mention that in two-loop order this formula has no regularization ambiguities, which only arise at three loop and beyond [13]. The advantage of eq.(5) for the evolution of the couplings is that it expresses the running of the gauge couplings in terms of the wavefunction renormalisation coefficients $Z_{\phi}$. Unlike the gauge wave function contribution which is one-loop exact, one still needs to use perturbation theory to compute the $Z_{\phi}$ order by order for the matter wave function. However, for a two loop approximation for the gauge couplings, only a one-loop calculation of $Z_{\phi}$ is required, simplifying the calculation. Their evolution from the unification scale where they are normalised to unity the heavy states is affected by the presence of $n=\left(n_{5}+3 n_{10}\right) / 2$ pairs of complete $S U(5)$ multiplets which affects the anomalous dimensions of the matter fields?.

Below the supersymmetry breaking scale we have to include the effect of the low energy supersymmetric thresholds. Their effect at one-loop, which corresponds to a two-loop effect if the splitting of the super-partner masses is of radiative origin, we denote by $\delta_{i}$. This term is needed because eq. (5) is valid only as long as supersymmetry is unbroken $\$$.

\footnotetext{
${ }^{5}$ These exotic Higgs effects will not be considered further in this paper

${ }^{6} Z_{\phi}$ depend on the unification scale!

${ }^{7}$ The running of the gauge couplings depends on $n_{5}+3 n_{10}$ only and not separately on $n_{5}$ or $n_{10}$ [1].

${ }^{8} \delta_{i}$ include regularisation scheme conversion factors as well.
} 
With these considerations we rewrite eq. (5) for the three gauge couplings ( $\mathrm{j}$ is a generation index) in the following way:

$$
\begin{aligned}
\alpha_{i}^{-1}\left(M_{z}\right)= & -\delta_{i}+\alpha_{g}^{-1}+\frac{b_{i}}{2 \pi} \ln \frac{M_{g}}{M_{z}}+\frac{n}{2 \pi} \ln \frac{M_{g}}{\mu_{g}}-\frac{\beta_{i, H_{1}}}{2 \pi} \ln Z_{H_{1}}\left(M_{z}\right)-\frac{\beta_{i, H_{2}}}{2 \pi} \ln Z_{H_{2}}\left(M_{z}\right) \\
& -\frac{\beta_{i, g}}{2 \pi} \ln \left[\frac{\alpha_{g}}{\alpha_{i}\left(M_{z}\right)}\right]^{1 / 3}-\sum_{j=1}^{3} \sum_{\phi_{j}} \frac{\beta_{i, \phi_{j}}}{2 \pi} \ln Z_{\phi_{j}}\left(M_{z}\right)
\end{aligned}
$$

where $b_{1}=33 / 5, b_{2}=1, b_{3}=-3$ and where $\beta_{i, \phi_{j}} \equiv T\left(R_{\phi_{j}}^{i}\right), i=\{1,2,3\}$, are the contributions to oneloop beta function 19 of the matter fields $\phi_{j}$ ( $\mathrm{j}=$ generation index), while $\beta_{i, g} \equiv T^{i}(G)$ is the one-loop beta function for the pure gauge (+gaugino) sector; the Higgs (+higgsino) sector contribution is included separately via the terms proportional to $\beta_{i, H 1,2}$ considered. Thus we have

$$
\beta_{i, \phi_{j}}=\left(\begin{array}{ccccc}
\frac{3}{10} & \frac{1}{10} & \frac{3}{5} & \frac{4}{5} & \frac{1}{5} \\
\frac{1}{2} & \frac{3}{2} & 0 & 0 & 0 \\
0 & 1 & 0 & \frac{1}{2} & \frac{1}{2}
\end{array}\right)_{i, \phi_{j}} \quad \beta_{i, g}=\left(\begin{array}{c}
0 \\
-6 \\
-9
\end{array}\right) ; \quad \beta_{i, H_{1,2}}=\left(\begin{array}{c}
\frac{3}{10} \\
\frac{1}{2} \\
0
\end{array}\right)
$$

independent of the values of $j$. The field $\phi_{j}$ runs over the set $\phi_{j}=\left\{l_{L}, q_{L}, e_{R}, u_{R}, d_{R}\right\}_{j}$, in this order, with $j$ as generation index.

\section{$3.1 \alpha_{3}\left(M_{Z}\right), M_{g}$ and $\mu_{g}$.}

To compute the two-loop values for the strong coupling at electroweak scale, the unification scale and the decoupling scale $\mu_{g}$ of the extra massive states $\square$, we use the two step method developed in reference 迎.

The first step eliminates the low energy supersymmetric thresholds dependence by expressing our results as a change to MSSM predictions for the strong coupling and the unification scale; this is possible because, to two loop order for the RGE, the $\delta_{i}$ 's are the same in both cases. Note that this means that our results will be expressed in terms of $\alpha_{g}^{o}, M_{g}^{o}$ and $\alpha_{i}^{o}\left(M_{z}\right)$ which all have the $\delta_{i}$ dependence included.

The second step is more technical and consists of replacing the arguments of the two-loop log's by the one-loop approximation. This will, of course, generate the evolution of the couplings correct to two-loop order.

To implement the first step note that in the MSSM we have

$$
\begin{aligned}
\alpha_{i}^{o-1}\left(M_{z}\right)= & -\delta_{i}+\alpha_{g}^{o-1}+\frac{b_{i}}{2 \pi} \ln \frac{M_{g}^{o}}{M_{z}}-\frac{\beta_{i, H_{1}}}{2 \pi} \ln Z_{H_{1}}^{o}\left(M_{z}\right)-\frac{\beta_{i, H_{2}}}{2 \pi} \ln Z_{H_{2}}^{o}\left(M_{z}\right) \\
& -\frac{\beta_{i, g}}{2 \pi} \ln \left[\frac{\alpha_{g}^{o}}{\alpha_{i}^{o}\left(M_{z}\right)}\right]^{1 / 3}-\sum_{j=1}^{3} \sum_{\phi_{j}} \frac{\beta_{i, \phi_{j}}}{2 \pi} \ln Z_{\phi_{j}}^{o}\left(M_{z}\right)
\end{aligned}
$$

where we employed the index "o" to label all MSSM related quantities, to distinguish them from those of the extended model which introduces additional heavy states. Note that $Z^{o}$ are normalised to unity at the $M_{g}^{o}, Z^{o}\left(M_{g}^{o}\right) \equiv Z^{o}(0)=1$.

\footnotetext{
${ }^{9}$ We also used that the one-loop beta function is $b=-3 T(G)+\sum T\left(R_{\phi}\right)$, where the sum runs over all chiral supermultiplets in representation $R_{\phi}$.

${ }^{10} \mu_{g}$ is the decoupling scale only in two loop order
} 
As is commonly done in all "bottom-up" approaches, we impose the conditions $\alpha_{1}\left(M_{z}\right)=\alpha_{1}^{o}\left(M_{z}\right)$ and $\alpha_{2}\left(M_{z}\right)=\alpha_{2}^{o}\left(M_{z}\right)$ and equal to their experimental values. We now determine the change of the strong coupling at electroweak scale; from (6), (8) we get that $(i=1,2)$ :

$$
\begin{aligned}
0= & \alpha_{g}^{-1}-\alpha_{g}^{o-1}+\frac{b_{i}}{2 \pi} \ln \frac{M_{g}}{M_{g}^{o}}+\frac{n}{2 \pi} \ln \frac{M_{g}}{\mu_{g}}-\frac{\beta_{i, H_{1}}}{2 \pi} \ln \left[\frac{Z_{H_{1}}\left(M_{z}\right)}{Z_{H_{1}}^{o}\left(M_{z}\right)}\right]-\frac{\beta_{i, H_{2}}}{2 \pi} \ln \left[\frac{Z_{H_{1}}\left(M_{z}\right)}{Z_{H_{1}}^{o}\left(M_{z}\right)}\right] \\
& -\frac{\beta_{i, g}}{2 \pi} \ln \left[\frac{\alpha_{g}}{\alpha_{g}^{o}}\right]^{1 / 3}-\sum_{j=1}^{3} \sum_{\phi_{j}} \frac{\beta_{i, \phi_{j}}}{2 \pi} \ln \left[\frac{Z_{\phi_{j}}\left(M_{z}\right)}{Z_{\phi_{j}}^{o}\left(M_{z}\right)}\right] \\
\delta \alpha_{3}^{-1}\left(M_{z}\right)= & \alpha_{g}^{-1}-\alpha_{g}^{o-1}+\frac{b_{3}}{2 \pi} \ln \frac{M_{g}}{M_{g}^{o}}+\frac{n}{2 \pi} \ln \frac{M_{g}}{\mu_{g}}-\frac{\beta_{3, H_{1}}}{2 \pi} \ln \left[\frac{Z_{H_{1}}\left(M_{z}\right)}{Z_{H_{1}}^{o}\left(M_{z}\right)}\right]-\frac{\beta_{3, H_{2}}}{2 \pi} \ln \left[\frac{Z_{H_{2}}\left(M_{z}\right)}{Z_{H_{2}}^{o}\left(M_{z}\right)}\right] \\
& -\frac{\beta_{3, g}}{2 \pi} \ln \left[\frac{\alpha_{g} \alpha_{3}^{o}\left(M_{z}\right)}{\alpha_{g}^{o} \alpha_{3}\left(M_{z}\right)}\right]^{1 / 3}-\sum_{j=1}^{3} \sum_{\phi_{j}} \frac{\beta_{3, \phi_{j}}}{2 \pi} \ln \left[\frac{Z_{\phi_{j}}\left(M_{z}\right)}{Z_{\phi_{j}}^{o}\left(M_{z}\right)}\right]
\end{aligned}
$$

where by $\delta \alpha_{3}^{-1}\left(M_{z}\right)$ we denoted the (two-loop induced) difference $\delta \alpha_{3}^{-1}\left(M_{z}\right)=1 / \alpha_{3}\left(M_{z}\right)-1 / \alpha_{3}^{o}\left(M_{z}\right)$. We note that the term $\ln \left(\alpha_{3}^{o}\left(M_{z}\right) / \alpha_{3}\left(M_{z}\right)\right.$ can simply be neglected in the two-loop approximation as $\alpha_{3}^{o}\left(M_{z}\right)$ and $\alpha_{3}\left(M_{z}\right)$ are equal in one-loop, and therefore this term would bring a higher order correction. We solve the system of eqs. (9), (10) for $\delta \alpha_{3}^{-1}\left(M_{z}\right), M_{g}$ and $\mu_{g}$ in function of $\alpha_{g}$ to get:

$$
\delta \alpha_{3}^{-1}\left(M_{z}\right)=-\frac{\sigma_{g}}{2 \pi} \ln \left[\frac{\alpha_{g}}{\alpha_{g}^{o}}\right]^{\frac{1}{3}}-\sum_{j=1}^{3} \sum_{\phi_{j}} \frac{\sigma_{\phi_{j}}}{2 \pi} \ln \left[\frac{Z_{\phi_{j}}\left(M_{z}\right)}{Z_{\phi_{j}}^{o}\left(M_{z}\right)}\right]-\frac{\sigma_{H_{1}}}{2 \pi} \ln \left[\frac{Z_{H_{1}}\left(M_{z}\right)}{Z_{H_{1}}^{o}\left(M_{z}\right)}\right]-\frac{\sigma_{H_{2}}}{2 \pi} \ln \left[\frac{Z_{H_{2}}\left(M_{z}\right)}{Z_{H_{2}}^{o}\left(M_{z}\right)}\right]
$$

where $\sigma_{\phi_{j}}=\beta_{1, \phi_{j}}\left(b_{2}-b_{3}\right) /\left(b_{1}-b_{2}\right)+\beta_{2, \phi_{j}}\left(b_{3}-b_{1}\right) /\left(b_{1}-b_{2}\right)+\beta_{3, \phi_{j}}$ and we have a similar definition for $\sigma_{H_{1}}, \sigma_{H_{2}}$ and $\sigma_{g}$. Thus

$$
\sigma=\left\{l_{L_{j}}: \frac{-9}{14}, q_{L_{j}}: \frac{-3}{2}, H_{1}: \frac{-9}{14}, H_{2}: \frac{-9}{14}, e_{R_{j}}: \frac{3}{7}, u_{R_{j}}: \frac{15}{14}, d_{R_{j}}: \frac{9}{14}, g: \frac{9}{7}\right\}
$$

We observe that $\sigma_{\phi_{j}}$ is negative for $S U(2)$ doublets and positive for $S U(2)$ singlets and these will therefore have opposite effects on the values of $\alpha_{3}\left(M_{z}\right)$, for same sign of the associated logarithmic factor. Moreover, the contribution to the strong coupling depends on whether $Z$ 's are larger or smaller than unity, which is determined by the relative effect of gauge and Yukawa contributions to their running. This should become clearer later. The overall effect on the strong coupling depends however on the relative magnitude of the wavefunction coefficients, $Z$, of the extended model compared to those of MSSM, $Z^{\circ}$.

Using the notation $\Delta \beta_{12, \phi_{j}}=\beta_{1, \phi_{j}}-\beta_{2, \phi_{j}}$ and $\Delta \beta_{12, H_{1,2}}=\beta_{1, H_{1,2}}-\beta_{2, H_{1,2}}$ the change in the unification scale factor is given by (see eqs.(9), (10)):

$$
\ln \frac{M_{g}}{M_{g}^{o}}=\frac{15}{14} \ln \left[\frac{\alpha_{g}}{\alpha_{g}^{o}}\right]^{\frac{1}{3}}+\frac{5}{28} \sum_{j=1}^{3} \sum_{\phi_{j}} \Delta \beta_{12, \phi_{j}} \ln \left[\frac{Z_{\phi_{j}}\left(M_{z}\right)}{Z_{\phi_{j}}^{o}\left(M_{z}\right)}\right]+\left\{\frac{5}{28} \Delta \beta_{12, H_{1}} \ln \left[\frac{Z_{H_{1}}\left(M_{z}\right)}{Z_{H_{1}}^{o}\left(M_{z}\right)}\right]+H_{1} \leftrightarrow H_{2}\right\}
$$

Note that $\Delta \beta_{12, \phi_{j}}$ has negative sign for $S U(2)$ doublets and positive for $S U(2)$ singlets (just like $\sigma_{\phi_{j}}$ ) and hence, for same sign of the logarithmic factor, these terms will drive the unification scale in opposite directions. Moreover, comparing eqs.(11) and (13), we observe that the unification scale $M_{g}$ is increased for positive $\Delta \beta_{12}$ eq.(13), while alpha strong eq.(11) is also increased巴⿴囗十 for a given (positive) sign of the

\footnotetext{
${ }^{11} \Delta \beta_{12, \phi_{j}}$ has same sign as $\sigma_{\phi_{j}}$
} 
log's. Thus, we already see it is difficult to decrease the strong coupling and increase unification scale simultaneously. One could argue that $Z$ factors have an implicit unification scale dependence as well, and that our above explanation does not apply; however this dependence comes in under the log, and is therefore very mild. Note that this applies independent of the type of Yukawa interaction, implicitly present in the values of $Z$ coefficients. This seems to be generic to models which consider complete additional representations and it was also explored in [1] in the absence of Yukawa effects with same conclusions. The above discussion should become clearer later when a more quantitative study will be made.

Finally, the decoupling scale $\mu_{g}$ is given by (see eqs.(9), (100):

$\ln \frac{\mu_{g}}{M_{g}^{o}}=\frac{2 \pi}{n}\left[\frac{1}{\alpha_{g}}-\frac{1}{\alpha_{g}^{o}}\right]+\frac{\Omega_{g}}{n} \ln \left[\frac{\alpha_{g}}{\alpha_{g}^{o}}\right]^{\frac{1}{3}}+\sum_{j=1}^{3} \sum_{\phi_{j}} \frac{\Omega_{\phi_{j}}}{n} \ln \left[\frac{Z_{\phi_{j}}\left(M_{z}\right)}{Z_{\phi_{j}}^{o}\left(M_{z}\right)}\right]+\left\{\frac{\Omega_{H_{1}}}{n} \ln \left[\frac{Z_{H_{1}}\left(M_{z}\right)}{Z_{H_{1}}^{o}\left(M_{z}\right)}\right]+H_{1} \leftrightarrow H_{2}\right\}$

where

$$
\Omega_{F}=\frac{b_{2}^{\prime} \beta_{1, F}-b_{1}^{\prime} \beta_{2, F}}{b_{1}-b_{2}} ; \quad \Omega_{g}=\frac{b_{2}^{\prime} \beta_{1, g}-b_{1}^{\prime} \beta_{2, g}}{b_{1}-b_{2}} ;
$$

with $F \equiv\left\{\phi_{j}, H_{1}, H_{2}\right\}$ and $b_{i}^{\prime}=b_{i}+n$.

To simplify our expressions further we need to compute the wavefunction renormalisation coefficients. Above the mass of the additional states in the extended model the running of these coefficients is altered implying that the anomalous dimensions of the matter fields are changed. We have (with $t=1 /(2 \pi) \ln ($ scale $))$

$$
\frac{d}{d t} \ln Z_{F}(t) \equiv-4 \pi \gamma_{F}=2 \sum_{k=1}^{3} C_{k}(F) \alpha_{k}(t)+\sum_{\nu=\tau, b, t} \tilde{\mathcal{A}}_{\nu}(F) y_{\nu}(t)+\mathcal{O}\left(\alpha^{2}\right)
$$

In the above equation $C_{j}(F)$ is the second order Casimir operator for the field $F$ for the group $S U(j)$ and is generation independent. The coefficients $\tilde{\mathcal{A}}_{\nu}(F)$ depend on the type of the superpotential of the model. For various cases we consider, their values are given in the Appendix.

The above equation can easily be integrated to give full analytical expressions for the wavefunction coefficients, valid in one-loop approximation which is consistent with a two-loop running for the gauge couplings. The solution is of the type:

$$
Z_{F}(t)=Z_{F}^{G}(t) \times Z_{F}^{Y}(t)
$$

where $Z_{F}^{G}$ and $Z_{F}^{Y}$ are given by the gauge and Yukawa running parts respectively, with $Z_{F}^{G}(0)=1$ and $Z_{F}^{Y}(0)=1$. $Z_{F}^{G}$ is determined by the gauge group; for the extended model we get from eq.(16) and (117) that

$$
\begin{aligned}
Z_{F}^{G}\left(M_{z}\right) & =\prod_{k=1}^{3}\left[\frac{\alpha_{g}}{\alpha_{k}\left(\mu_{g}\right)}\right]^{-\frac{2 C_{k}(F)}{b_{k}^{\prime}}}\left[\frac{\alpha_{k}\left(\mu_{g}\right)}{\alpha_{k}\left(M_{z}\right)}\right]^{-\frac{2 C_{k}(F)}{b_{k}}} \\
& =\prod_{k=1}^{3}\left[\frac{\alpha_{g}}{\alpha_{k}\left(\mu_{g}\right)}\right]^{\frac{2 C_{k}(F)}{b_{k}} \frac{n}{b_{k}^{\prime}}}\left[\frac{\alpha_{g}}{\alpha_{k}\left(M_{z}\right)}\right]^{-\frac{2 C_{k}(F)}{b_{k}}}
\end{aligned}
$$

Note that for one-loop running of the coefficients $Z$ (consistent with two loop approximation for gauge couplings), the decoupling scale of the heavy states $\psi$ can be considered to be $\mu_{g}$ rather than a physical mean mass of the multiplet, as the difference is a radiative effect and therefore represents a higher (two-loop) correction to $Z$ 's or three loop order for the gauge couplings. This justifies the last equation we wrote above. 
Similarly, we have that in MSSM,

$$
Z_{F}^{o G}\left(M_{z}\right)=\prod_{k=1}^{3}\left[\frac{\alpha_{g}^{o}}{\alpha_{k}^{o}\left(M_{z}\right)}\right]^{-\frac{2 C_{k}(F)}{b_{k}}}
$$

The coefficients $Z_{F}^{Y}$ can also be expressed in a form similar to that of $Z_{F}^{G}$ (eq. (18)), in terms of Yukawa and gauge couplings at initial and final scale only; to do this one only needs the one-loop running for Yukawa couplings; from this, any Yukawa coupling can be expressed (see Appendix) in terms of the derivative of $\ln y_{\tau, b, t}$ and derivative of $\ln \alpha_{i}$ where the latter is obtained by replacing $\alpha_{i}$ with $\left(1 / \tilde{b}_{i}\right) d \ln \alpha_{i} / d t$, consistent with one-loop running for Yukawa; hence, the integral $\int y_{\nu} d t$ can be performed analytically and therefore the differential equation of $Z_{F}^{Y}$ 's (similar to (16) without the gauge term) can be integrated to give $Z_{F}^{Y}$ 's in one-loop approximation.

A final comment is in order here: we see that the powers entering eq.(18) depend on $n$ as $n /\left(b_{i}+n\right)$. This means that in the limit of large $n$ the contribution of these terms to the running of the couplings brings in only a small depedence on $\mathrm{n}$. We will later see that $\alpha_{g} / \alpha_{k}\left(\mu_{g}\right)$ itself is also relatively stable with respect to $n$. Next, from the running of the gauge couplings eqs. (5) and (6) we also observe that the explicit $n$ dependence comes in under same form for all the couplings as $n /(2 \pi) \ln \left(M_{g} / \mu_{g}\right)$, which means that the relative behaviour of the gauge couplings evolution is affected only through $Z$ factors. With $\alpha_{1}\left(M_{z}\right)$ and $\alpha_{2}\left(M_{z}\right)$ fixed to their experimental values, and the above observations we conclude that the predictions for the strong coupling at electroweak scale will be stable for large $n$. This observation applies to other predictions we will make as well, such as the unification scale and the bare mass, $\mu_{g}$. For the absence of Yukawa couplings' effects this behaviour was shown in [1]. We will see that this remains true in their presence too, although a dependence of the particular type of superpotential might be expected.

\subsection{Analysis for 3rd generation Yukawa couplings only.}

We will consider first the case when the superpotential is the same in both MSSM and in the extended model. In eqs. (11), (13), (14), we replace the gauge part of $Z$ coefficients, $Z_{F}^{G}$, with their one-loop analytical expressions, consistent with two-loop running for the gauge couplings. For the Yukawa part of $Z$ coefficients, $Z_{F}^{Y}$, from their evolution equation

$$
\frac{d}{d t} \ln Z_{F}^{Y}(t)=\sum_{\nu=\tau, b, t} \mathcal{A}_{\nu}(F) y_{\nu}(t)+\mathcal{O}\left(\alpha^{2}\right)
$$

after using the relations among the coefficients $\mathcal{A}_{\nu}(F)$, (not affected by the presence of the extra matter $^{12}$ ), one can get the following relations, valid at any scale between $M_{g}$ and $M_{z}$

$$
\begin{aligned}
Z_{Q_{3}}^{Y} & =\left(Z_{b_{R}}^{Y} Z_{t_{R}}^{Y}\right)^{1 / 2} \\
Z_{L_{3}}^{Y} & =Z_{\tau_{R}}^{{ }^{1 / 2}} \\
Z_{H_{1}}^{Y} & =Z_{b_{R}}^{{ }^{3 / 2}} Z_{\tau_{R}}^{Y}{ }^{1 / 2} \\
Z_{H_{2}}^{Y} & =Z_{t_{R}}^{3^{3 / 2}}
\end{aligned}
$$

where we used, without any loss of generality, the convention $Z^{Y}(0)=1$ for any field. Making use of these relations, we get from eq.(11) that

$$
\delta \alpha_{3}^{-1}\left(M_{z}\right)=-\frac{470}{77 \pi} \ln \left[\frac{\alpha_{g}}{\alpha_{g}^{o}}\right]+\sum_{j=1}^{3} \ln \left\{1+\frac{b_{j}^{\prime}}{n}\left[\frac{\alpha_{g}}{\alpha_{g}^{o}}-1\right]\right\}^{\omega_{j}}
$$

\footnotetext{
${ }^{12}$ Their expressions are presented in Appendix
} 


$$
+\frac{3}{28 \pi}\left[5 \ln \frac{Z_{b_{R}}^{Y}}{Z_{b_{R}}^{o Y}}+\ln \frac{Z_{\tau_{R}}^{Y}}{Z_{\tau_{R}}^{o Y}}+3 \ln \frac{Z_{t_{R}}^{Y}}{Z_{t_{R}}^{o Y}}\right]
$$

where $Z^{Y}, Z^{o Y}$ factors are evaluated at $M_{z}$ scale and where we defined

$$
\omega_{j}=\left\{\frac{-2}{11 \pi} \frac{n}{b_{1}^{\prime}}, \frac{81}{14 \pi} \frac{n}{b_{2}^{\prime}}, \frac{2}{7 \pi} \frac{n}{b_{3}^{\prime}}\right\}_{j}
$$

and $\delta \alpha_{3}^{-1}\left(M_{z}\right)=\alpha_{3}^{-1}\left(M_{z}\right)-\alpha_{3}^{o-1}\left(M_{z}\right)$. To get the above formula we replaced the terms of the form $\ln \left(\alpha_{g} / \alpha_{j}\left(\mu_{g}\right)\right)$ by

$$
\ln \left[\frac{\alpha_{g}}{\alpha_{j}\left(\mu_{g}\right)}\right]=\ln \left[1+\alpha_{g} \frac{b_{j}^{\prime}}{2 \pi} \ln \left(\frac{M_{g}}{\mu_{g}}\right)\right]=\ln \left[1+\frac{b_{j}^{\prime}}{n}\left(\frac{\alpha_{g}}{\alpha_{g}^{o}}-1\right)\right]
$$

where we made use of the fact that $\alpha_{g}^{-1}-\alpha_{g}^{o-1}+n /(2 \pi) \ln \left(M_{g} / \mu_{g}\right)=0$ in one-loop order $[3$ which is obtained from equations (9) and (10) after ${ }^{4}$ noticing that, in one-loop approximation, all wavefunction renormalisation coefficients are equal to 1.

Similar results hold for the unification scale and for the decoupling scale of the extra matter. We have from eqs.(13), (21)

$$
\frac{M_{g}}{M_{g}^{o}}=\left[\frac{\alpha_{g}}{\alpha_{g}^{o}}\right]^{\frac{31}{21}} \prod_{j=1}^{3}\left\{1+\frac{b_{j}^{\prime}}{n}\left[\frac{\alpha_{g}}{\alpha_{g}^{o}}-1\right]\right\}^{\rho_{j}}\left[\frac{Z_{b_{R}}^{o Y}}{Z_{b_{R}}^{Y}}\right]^{\frac{1}{7}}\left[\frac{Z_{\tau_{R}}^{Y}}{Z_{\tau_{R}}^{o Y}}\right]^{\frac{1}{14}}\left[\frac{Z_{t_{R}}^{o Y}}{Z_{t_{R}}^{Y}}\right]^{\frac{1}{28}}
$$

where $Z^{Y}, Z^{o Y}$ factors are evaluated at $M_{z}$ scale and

$$
\rho_{j}=\left\{\frac{1}{12} \frac{n}{b_{1}^{\prime}}, \frac{-39}{28} \frac{n}{b_{2}^{\prime}}, \frac{4}{21} \frac{n}{b_{3}^{\prime}}\right\}_{j}
$$

Finally from eq.(14) and (21)

$$
\frac{\mu_{g}}{M_{g}^{o}}=\left[\frac{\alpha_{g}}{\alpha_{g}^{o}}\right]^{\frac{31}{21}+\frac{2336}{231 n}} \exp \left[\frac{2 \pi}{n}\left(\alpha_{g}^{-1}-\alpha_{g}^{o-1}\right)\right] \prod_{j=1}^{3}\left\{1+\frac{b_{j}^{\prime}}{n}\left[\frac{\alpha_{g}}{\alpha_{g}^{o}}-1\right]\right\}^{\sigma_{j}}\left[\frac{Z_{\tau_{R}}^{Y}}{Z_{\tau_{R}}^{O Y}}\right]^{r_{1}}\left[\frac{Z_{b_{R}}^{Y}}{Z_{b_{R}}^{o Y}}\right]^{r_{2}}\left[\frac{Z_{t_{R}}^{Y}}{Z_{t_{R}}^{o Y}}\right]^{r_{3}}
$$

with

$$
r_{1}=\frac{1}{14}-\frac{3}{7 n}, \quad r_{2}=\frac{-1}{7}-\frac{23}{14 n}, \quad r_{3}=\frac{-1}{28}-\frac{43}{28 n}
$$

and

$$
\sigma_{j}=\left\{\frac{11 n-7}{132 b_{1}^{\prime}}, \frac{-3(111+13 n)}{28 b_{2}^{\prime}}, \frac{4(22+n)}{21 b_{3}^{\prime}}\right\}_{j}
$$

In the above expressions eqs.(22), (25), (27), $Z^{Y}$ factors are evaluated at the electroweak scale, and normalised to unity at the unification scale $M_{g}$ and the same is true for the $Z^{o}$ coefficients ${ }^{\boxplus}$. If we set all "Yukawa" wavefunction coefficients $Z_{F}^{Y}$ and $Z_{F}^{o Y}$ equal to unity, we recover the results [1] we obtained previously in the absence of any Yukawa coupling:

\footnotetext{
${ }^{13} \operatorname{In}$ fact $\alpha_{g}^{-1}-\alpha_{g}^{o-1}+b_{i} /(2 \pi) \ln \left(M_{g} / M_{g}^{o}\right)+n /(2 \pi) \ln \left(M_{g} / \mu_{g}\right)=0$ in one loop and we further have $\ln \left(M_{g} / M_{g}^{o}\right)=0$ in one-loop because the change of unification scale is a two-loop effect.

${ }^{14} \mathrm{We}$ also have that $\delta \alpha_{3}^{-1}$ is non-zero in two-loop only, being 0 in one-loop.

${ }^{15}$ These are normalised to unity at $M_{g}^{o}$ !

${ }^{16}$ In ref. [1] the results of the extended model were compared with those of MSSM without top, bottom, tau Yukawa effects.
} 
$Z_{F}^{G} \leq 1$ ) (see eqs.(16), (20)), we note that their effect is to decrease the strong coupling at electroweak scale. However, we must evaluate the relative effect of these coefficients $Z^{Y}$ to those of MSSM, $Z^{o Y}$; this will be done below, by expressing them in terms of Yukawa couplings, assuming the same low energy input for Yukawa couplings in both EMSSM and MSSM models. This means we take same values at the electroweak scale for top, bottom and $\tau$ Yukawa couplings in both models. Using the expressions of $Z^{Y}$ and $Z^{o Y}$, the result will be expressed as a function of ratios of the Yukawa couplings, following the details presented at the end of the previous subsection, after eq.(19).

For the strong coupling eq.(22) we obtain the following result: $1 / \alpha_{3}\left(M_{z}\right)=1 / \alpha_{3}^{o}\left(M_{z}\right)+\delta \alpha_{3}^{-1}\left(M_{z}\right)$ with

$$
\begin{aligned}
\delta \alpha_{3}^{-1}\left(M_{z}\right)= & -\frac{11563}{2013 \pi} \ln \left[\frac{\alpha_{g}}{\alpha_{g}^{o}}\right]+\sum_{j=1}^{3} \ln \left\{1+\frac{b_{j}^{\prime}}{n}\left[\frac{\alpha_{g}}{\alpha_{g}^{o}}-1\right]\right\}^{\mathcal{U}_{j}} \\
& +\frac{3}{854 \pi}\left\{4 \ln \left[\frac{y_{\tau}(0)}{y_{\tau}\left(M_{z}\right)}\right]+45 \ln \left[\frac{y_{b}(0)}{y_{b}\left(M_{z}\right)}\right]+23 \ln \left[\frac{y_{t}(0)}{y_{t}\left(M_{z}\right)}\right]\right\} \\
& -\frac{3}{854 \pi}\left\{4 \ln \left[\frac{y_{\tau}^{o}(0)}{y_{\tau}^{o}\left(M_{z}\right)}\right]+45 \ln \left[\frac{y_{b}^{o}(0)}{y_{b}^{o}\left(M_{z}\right)}\right]+23 \ln \left[\frac{y_{t}^{o}(0)}{y_{t}^{o}\left(M_{z}\right)}\right]\right\}
\end{aligned}
$$

and

$$
\mathcal{U}_{j}=\left\{\frac{-2923}{14091 \pi} \frac{n}{b_{1}^{\prime}}, \frac{4293}{854 \pi} \frac{n}{b_{2}^{\prime}}, \frac{130}{183 \pi} \frac{n}{b_{3}^{\prime}}\right\}_{j}
$$

$j=\{1,2,3\}$. The terms which involve $\alpha_{g} / \alpha_{g}^{o}$ in the r.h.s. of eq.(30) drive $\alpha_{3}\left(M_{z}\right)$ above MSSM value. We also see that, imposing the same electroweak scale values for Yukawa couplings in both models, the overall effect of the Yukawa couplings depends on $\ln \left(y_{\tau}(0) / y_{\tau}^{o}(0)\right), \ln \left(y_{b}(0) / y_{b}^{o}(0)\right)$ and $\ln \left(y_{t}(0) / y_{t}^{o}(0)\right)$. The contribution of these terms is negative and hence $\alpha_{3}\left(M_{z}\right)$ is further increased from its MSSM value. The reason for this is that the running of Yukawa couplings is similar in both models $\square$, but in the extended model they decrease faster due to the fact the gauge couplings in EMSSM at any scale are increased from their corresponding MSSM values. This means that, for a fixed low energy input for Yukawa couplings and $S U(2)$ and $U(1)$ gauge couplings, the net effect due the presence of the extra multiplets is to decrease the Yukawa couplings at unification scale while increasing the value of the unified coupling. Hence $y(0)<y^{o}(0)$ for top, bottom and tau quarks and $\alpha_{3}\left(M_{z}\right)$ is increased. Note that this is true in the case we have the same superpotential in both models which means that the coefficients in front of Yukawa couplings in their one loop running equations are the same to those of MSSM.

The Yukawa effects come in eq. (30) as log's of ratios $y_{\lambda}(0) / y_{\lambda}(Q)$ with positive sign in front; this might no longer be true if the superpotential of the model we consider for the extended model is changed. Still, it seems that a domain with large initial Yukawa couplings $y_{\lambda}(0)$ would favour a decreasing effect on the strong coupling at electroweak scale, particularly when they are larger than their electroweak scale values. This would in turn favour a fast rate of approach towards infrared fixed points for ratios of Yukawa to any gauge coupling [8]. However, getting a large initial Yukawa coupling, with same low energy input values as in MSSM is not easy, due to, as we mentioned it above, the gauge couplings competing effect which seems to be difficult to avoid. In cases with unified coupling larger than in MSSM, as it happens in our model with and due to the extra heavy states, the gauge couplings increase while increasing the scale, causing the Yukawa couplings to decrease as we increase the scale. This could eventually be compensated for by increasing the values of the coefficients in front of Yukawa couplings in the one-loop running of Yukawa couplings. This is possible if the Yukawa structure is richer than in MSSM; such a particular case will be explored in the next subsection.

\footnotetext{
${ }^{17}$ i.e.they have the same anomalous dimension coefficients
} 
The unification scale is given by (see eq. 25$)$ )

$$
\frac{M_{g}}{M_{g}^{o}}=\left[\frac{\alpha_{g}}{\alpha_{g}^{o}}\right]^{\frac{1711}{1098}} \prod_{j=1}^{3}\left\{1+\frac{b_{j}^{\prime}}{n}\left[\frac{\alpha_{g}}{\alpha_{g}^{o}}-1\right]\right\}^{f_{j}} \prod_{\lambda=\tau, b, t}\left[\frac{y_{\lambda}(0)}{y_{\lambda}\left(M_{z}\right)} \frac{y_{\lambda}^{o}\left(M_{z}\right)}{y_{\lambda}^{o}(0)}\right]^{g_{\lambda}}
$$

where

$$
f_{j}=\left\{\frac{1133}{15372} \frac{n}{b_{1}^{\prime}}, \frac{-2277}{1708} \frac{n}{b_{2}^{\prime}}, \frac{32}{549} \frac{n}{b_{3}^{\prime}}\right\}_{j}
$$

and

$$
g_{\lambda}=\left\{\frac{93}{1708}, \frac{-128}{1708}, \frac{1}{1708}\right\}_{\lambda}
$$

with $\lambda=\{\tau, b, t\}$ in this order. The presence of the factor

$$
K=\prod_{\lambda=\tau, b, t}\left[\frac{y_{\lambda}(0)}{y_{\lambda}\left(M_{z}\right)} \frac{y_{\lambda}^{o}\left(M_{z}\right)}{y_{\lambda}^{o}(0)}\right]^{g_{\lambda}}
$$

in the expression for $M_{g} / M_{g}^{o}$ prevents us from simplifying any further this analytical expression. To make predictions one needs, just as in the previous case, the initial value (at unification scale) of the Yukawa couplings and their value at the electroweak scale. Since the latter correspond to the masses of the third family it is reasonable to take them to be the same in the MSSM and in the extended model. Hence $K$ depends on $y_{\lambda}(0) / y_{\lambda}^{o}(0)$ only. This dependence is very mild however because the powers $g_{\lambda}$ are very small and therefore, with a good approximation we have that

$$
K \approx 1
$$

We checked this numerically $[8$ by using the one-loop running for Yukawa couplings with one-loop running for the gauge couplings for MSSM case and for the extended model (in the presence of the extra-matter) and observed that the ratios $y_{\lambda}^{o}(0) / y_{\lambda}(0)$ are of order 10 giving $K=1-2$, with larger $K$ (up to 1.8) for larger unified coupling. The main contribution to increasing the value of $K$ comes from the bottom quark as $K$ contains a negative power of $y_{b}(0) / y_{b}^{o}(0) \leq 1$, giving an increase factor larger than unity, but still very small due to small $g_{\lambda}$, as mentioned (this is further suppressed by the tau' contribution). For $n=1,2,3$ case the same discussion about the validity of these results as that presented in [1] applies. Hence we have a clear prediction for the unification scale,

$$
\frac{M_{g}}{M_{g}^{o}} \approx\left[\frac{\alpha_{g}}{\alpha_{g}^{o}}\right]^{\frac{1711}{1098}} \prod_{j=1}^{3}\left\{1+\frac{b_{j}^{\prime}}{n}\left[\frac{\alpha_{g}}{\alpha_{g}^{o}}-1\right]\right\}^{f_{j}}
$$

This increase of the unification scale is very close to that found in reference [1] and, for $\alpha_{g} \leq 10 \alpha_{g}^{o} \approx 0.4$ is less than $\approx 3.5$, and depends on the values of $n$, with largest value for smallest $n$. For large $n$ the results are stable, as we previously mentioned. We conclude that the effects of Yukawa couplings on the scale are small and thus there is no need for a further numerical calculation.

Finally, the bare mass of heavy states is given by

$$
\frac{\mu_{g}}{M_{g}^{o}}=\left[\frac{\alpha_{g}}{\alpha_{g}^{o}}\right]^{\frac{1711}{1098}+\frac{104039}{12078 n}} \exp \left[\frac{2 \pi}{n}\left(\frac{1}{\alpha_{g}}-\frac{1}{\alpha_{g}^{o}}\right)\right] \prod_{j=1}^{3}\left\{1+\frac{b_{j}^{\prime}}{n}\left[\frac{\alpha_{g}}{\alpha_{g}^{o}}-1\right]\right\}^{\mathcal{R}_{j}} \prod_{\lambda=\tau, b, t}\left[\frac{y_{\lambda}(0)}{y_{\lambda}\left(M_{z}\right)} \frac{y_{\lambda}^{o}\left(M_{z}\right)}{y_{\lambda}^{o}(0)}\right]^{\mathcal{S}_{\lambda}}
$$

\footnotetext{
${ }^{18}$ For generic values for Yukawa couplings for high and low $\tan \beta$ case see ref. [14.
} 


$$
\begin{gathered}
\mathcal{R}_{j}=f_{j}+\left\{\frac{10909}{169092 b_{1}^{\prime}}, \frac{-15339}{1708 b_{2}^{\prime}}, \frac{1460}{549 b_{3}^{\prime}}\right\}_{j} \\
\mathcal{S}_{\lambda}=g_{\lambda}+\left\{\frac{-187}{1708 n}, \frac{-716}{1708 n}, \frac{-755}{1708 n}\right\}_{\lambda}
\end{gathered}
$$

with $j=\{1,2,3\}$ and $\lambda=\{\tau, b, t\}$. We again take Yukawa couplings at $M_{z}$ scale be equal in both MSSM and our extended model. The dependence of this $\mu_{g}$ scale on the Yukawa couplings $y_{\lambda}(0) / y_{\lambda}^{o}(0)$ is again weak for large $n$, and therefore the results stay close to those of reference [1]. We would like to note that our results for unification scale, the strong coupling and decoupling scale $\mu_{g}$ were all computed in terms of Yukawa couplings at the unification and electroweak scale. The values of these Yukawa couplings can be determined numerically from their one-loop running only, and we do not need any numerical work for the gauge couplings running, thus simplifying the calculation for $M_{g}, \alpha_{3}\left(M_{z}\right)$ and $\mu_{g}$.

\subsection{Family symmetric Yukawa couplings.}

The predictions we make for the strong coupling and the unification scale depend on the type of superpotential we assume; this is so because the running of Yukawa couplings (and therefore their effect on the running of the gauge couplings) depends on the type of superpotential. We considered in the previous section a MSSM type of superpotential and its predictions. We consider now a different superpotential, to show how these predictions change. The pattern of the results we present could prove helpful in designing models with better phenomenological predictions.

We thus turn now to a discussion of the implications of a model designed to give an acceptable structure for all quark and lepton masses including the light generations [7]. In this case the new superpotential has the following form:

$$
W=\sum_{i, j=1}^{3}\left(h_{i j}^{u} Q_{i} U_{j} H_{2}^{i j}+h_{i j}^{d} Q_{i} D_{j} H_{1}^{i j}+h_{i j}^{l} L_{i} e_{j} H_{1}^{i j}\right)
$$

where the structure in the light quark mass matrix is driven via mixing in the Higgs sector so that the two light Higgs doublets of the MSSM are mixtures of the Higgs doublets $H_{1}^{i j}$ and of $H_{2}^{i j}$. This form is able to reproduce the values of the masses of the third generation for the case the Yukawa couplings are given by their infra-red fixed point values in terms of the gauge couplings [8]. We use this as our starting point and take the fixed point values for the $h_{i j}^{u}$. This means they are all equal, independent

of $i, j$. Similarly we take $h_{i j}^{d}$ and $h_{i j}^{l}$ to be $i, j$ independent and at their fixed points. With this we find

$$
\frac{d}{d t} \ln Z_{F}^{Y}(t)=\sum_{\nu=\tau, b, t} \mathcal{B}_{\nu}(F) y_{\nu}(t)+\mathcal{O}\left(\alpha^{2}\right)
$$

where $\mathcal{B}_{\nu}(\mathcal{F})$ is presented in the Appendix. From eq. (42) we obtain the following relations, valid at any scale $\mathcal{M}$ larger than $\mu_{g}, \mathcal{M} \geq \mu_{g}$

$$
\begin{aligned}
Z_{Q_{j}}^{Y}(\mathcal{M}) & =\left[Z_{b_{R}}^{Y}(\mathcal{M}) Z_{t_{R}}^{Y}(\mathcal{M})\right]^{1 / 2} \\
Z_{L_{j}}^{Y}(\mathcal{M}) & =\left[Z_{\tau_{R}}^{Y}(\mathcal{M})\right]^{1 / 2} \\
Z_{H_{1}^{i j}}^{Y}(\mathcal{M}) & =\left[Z_{b_{R}}^{Y}(\mathcal{M})\right]^{1 / 2}\left[Z_{\tau_{R}}^{Y}(\mathcal{M})\right]^{1 / 6} \\
Z_{H_{2}^{i j}}^{Y}(\mathcal{M}) & =\left[Z_{t_{R}}^{Y}(\mathcal{M})\right]^{1 / 2}
\end{aligned}
$$


For the case $M_{Z} \leq \mathcal{M} \leq \mu_{g}$ the above type of superpotential is no longer valid and a MSSM-like superpotential applies. This is so because the extra heavy states we consider, among which are all Higgs fields 5 of eq. (41) decouple at $\mu_{g}$. Below this scale $\mu_{g}$, only the third generation' Yukawa couplings give important contributions to wavefunction renormalisation coefficients $Z_{\phi_{k}}^{Y}$, while for the first two generations the factors $Z_{\phi_{j}}, j=\{1,2\}$ evolve only through their gauge contributions, $Z_{\phi_{j}}^{G}, j=\{1,2\}$. We thus have that for $M_{Z} \leq \mathcal{M} \leq \mu_{g}$

$$
\frac{d}{d t} \ln Z_{F}^{Y}(t)=\sum_{\nu=\tau, b, t} \mathcal{A}_{\nu}(F) y_{\nu}(t)+\mathcal{O}\left(\alpha^{2}\right)
$$

which, after integration below $\mu_{g}$, gives that

$$
\begin{aligned}
& \frac{Z_{Q_{3}}^{Y}(\mathcal{M})}{Z_{Q_{3}}^{Y}\left(\mu_{g}\right)}=\left[\frac{Z_{b_{R}}^{Y}(\mathcal{M})}{Z_{b_{R}}^{Y}\left(\mu_{g}\right)} \frac{Z_{t_{R}}^{Y}(\mathcal{M})}{Z_{t_{R}}^{Y}\left(\mu_{g}\right)}\right]^{1 / 2} \\
& \frac{Z_{L_{3}}^{Y}(\mathcal{M})}{Z_{L_{3}}^{Y}\left(\mu_{g}\right)}=\left[\frac{Z_{\tau_{R}}^{Y}(\mathcal{M})}{Z_{\tau_{R}}^{Y}\left(\mu_{g}\right)}\right]^{1 / 2} \\
& \frac{Z_{H_{1}}^{Y}(\mathcal{M})}{Z_{H_{1}}^{Y}\left(\mu_{g}\right)}=\left[\frac{Z_{b_{R}}^{Y}(\mathcal{M})}{Z_{b_{R}}^{Y}\left(\mu_{g}\right)}\right]^{3 / 2}\left[\frac{Z_{\tau_{R}}^{Y}(\mathcal{M})}{Z_{\tau_{R}}^{Y}\left(\mu_{g}\right)}\right]^{1 / 2} \\
& \frac{Z_{H_{2}}^{Y}(\mathcal{M})}{Z_{H_{2}}^{Y}\left(\mu_{g}\right)}=\left[\frac{Z_{t_{R}}^{Y}(\mathcal{M})}{Z_{t_{R}}^{Y}\left(\mu_{g}\right)}\right]^{3 / 2}
\end{aligned}
$$

Note also the similarity with eq. (21), as expected, with the difference that the initial condition for any $Z^{Y}$ takes place at $\mu_{g}$ scale (with $Z^{Y}\left(\mu_{g}\right) \neq 1$ !) rather than at unification scale.

Following the procedure outlined above we obtain, in this case, the following change to the strong coupling:

$$
\begin{aligned}
\delta \alpha_{3}^{-1}\left(M_{z}\right)= & -\frac{470}{77 \pi} \ln \left[\frac{\alpha_{g}}{\alpha_{g}^{o}}\right]+\sum_{j=1}^{3} \ln \left\{1+\frac{b_{j}^{\prime}}{n}\left[\frac{\alpha_{g}}{\alpha_{g}^{o}}-1\right]\right\}^{\omega_{j}} \\
& +\frac{9}{28 \pi}\left[\ln Z_{b_{R}}^{Y}\left(\mu_{g}\right)-\ln Z_{t_{R}}^{Y}\left(\mu_{g}\right)-\frac{1}{3} \ln Z_{\tau_{R}}^{Y}\left(\mu_{g}\right)\right] \\
& +\frac{3}{28 \pi}\left[5 \ln \frac{Z_{b_{R}}^{Y}\left(M_{z}\right)}{Z_{b_{R}}^{Y}\left(\mu_{g}\right)}+\ln \frac{Z_{\tau_{R}}^{Y}\left(M_{z}\right)}{Z_{\tau_{R}}^{Y}\left(\mu_{g}\right)}+3 \ln \frac{Z_{t_{R}}^{Y}\left(M_{z}\right)}{Z_{t_{R}}^{Y}\left(\mu_{g}\right)}\right] \\
& -\frac{3}{28 \pi}\left[5 \ln Z_{b_{R}}^{o Y}\left(M_{z}\right)+\ln Z_{\tau_{R}}^{o Y}\left(M_{z}\right)+3 \ln Z_{t_{R}}^{o Y}\left(M_{z}\right)\right]
\end{aligned}
$$

where

$$
\omega_{j}=\left\{\frac{-2}{11 \pi} \frac{n}{b_{1}^{\prime}}, \frac{81}{14 \pi} \frac{n}{b_{2}^{\prime}}, \frac{2}{7 \pi} \frac{n}{b_{3}^{\prime}}\right\}_{j}
$$

and $\delta \alpha_{3}^{-1}\left(M_{z}\right)=\alpha_{3}^{-1}\left(M_{z}\right)-\alpha_{3}^{o-1}\left(M_{z}\right)$. Setting all $Z^{Y}$ equal to unity in (46) gives the previous result [1] where only gauge effects on $\alpha_{3}\left(M_{z}\right)$ were considered, leading to an increase of its value from the MSSM value. We also see that the Yukawa effects give in this case two negative signs in eq. (46) in the square bracket which make its contribution rather small and negative; For comparison see the result of eq. (22) for the MSSM superpotential. This contribution, if the signs were positive was expected to be the largest as $Z$ factors have a steeper running between $M_{g}$ (where they are equal to unity) and

\footnotetext{
${ }^{19}$ This implies that the number of extra heavy states is larger than 5
} 
$\mu_{g}$ than between $\mu_{g}$ and $M_{z}$ due to larger coefficients in (42) than in (44). The second-last and last square brackets in eq. (46) give a small correction to alpha strong and in fact they largely cancel each other. We can further express $\delta \alpha_{3}^{-1}\left(M_{z}\right)$ in function of Yukawa couplings evaluated at $M_{g}$, at $\mu_{g}$ and at electroweak scale.

$$
\begin{aligned}
\delta \alpha_{3}^{-1}\left(M_{z}\right)= & -\frac{11563}{2013 \pi} \ln \left[\frac{\alpha_{g}}{\alpha_{g}^{o}}\right]+\sum_{i=1}^{3} \mathcal{Z}_{j} \ln \left\{1+\frac{b_{j}^{\prime}}{n}\left[\frac{\alpha_{g}}{\alpha_{g}^{o}}-1\right]\right\} \\
& +\left\{\frac{243}{1022 \pi} \ln \left[\frac{y_{b}(0)}{y_{b}\left(\mu_{g}\right)}\right]-\frac{45}{511 \pi} \ln \left[\frac{y_{\tau}(0)}{y_{\tau}\left(\mu_{g}\right)}\right]-\frac{225}{1022 \pi} \ln \left[\frac{y_{t}(0)}{y_{t}\left(\mu_{g}\right)}\right]\right\} \\
& +\left\{\frac{135}{854 \pi} \ln \left[\frac{y_{b}\left(\mu_{g}\right)}{y_{b}\left(M_{z}\right)}\right]+\frac{6}{427 \pi} \ln \left[\frac{y_{\tau}\left(\mu_{g}\right)}{y_{\tau}\left(M_{z}\right)}\right]+\frac{69}{854 \pi} \ln \left[\frac{y_{t}\left(\mu_{g}\right)}{y_{t}\left(M_{z}\right)}\right]\right\} \\
& -\left\{\frac{135}{854 \pi} \ln \left[\frac{y_{b}^{o}(0)}{y_{b}^{o}\left(M_{z}\right)}\right]+\frac{6}{427 \pi} \ln \left[\frac{y_{\tau}^{o}(0)}{y_{\tau}^{o}\left(M_{z}\right)}\right]+\frac{69}{854 \pi} \ln \left[\frac{y_{t}^{o}(0)}{y_{t}^{o}\left(M_{z}\right)}\right]\right\}
\end{aligned}
$$

with $\mathcal{Z}_{j}$ given by

$$
\mathcal{Z}_{j}=\left\{\frac{1}{\pi}\left(\frac{-2923}{14091}+\frac{351}{365 b_{1}^{\prime}}\right), \frac{1}{\pi}\left(\frac{4293}{854}+\frac{-6129}{1022 b_{2}^{\prime}}\right), \frac{1}{\pi}\left(\frac{130}{183}+\frac{486}{511 b_{3}^{\prime}}\right)\right\}_{j}
$$

The terms in (48) which contain $\alpha_{g} / \alpha_{g}^{o}$ have an overall increasing effect on the strong coupling driving it towards values larger than in MSSM as it can be seen by simply plotting their sum with respect to $\alpha_{g}$. We must therefore analyse the effect Yukawa couplings in eq.(48) have. Their effect on $\alpha_{3}\left(M_{z}\right)$ is not always opposite to that of the gauge couplings. In the expression above we see three types of contributions. The first one is due to Yukawa effects between unification scale and (effective) decoupling scale $\mu_{g}$. This is due to the family symmetric Yukawa couplings we chose above this scale. We can assume that the values of $y_{\lambda}(0)$ are larger than $y_{\lambda}\left(\mu_{g}\right)$; this would be expected given that we considered the family symmetric couplings, which is possible if they flow to infrared fixed point values, thing favoured by a large initial Yukawa? The overall effect would therefore be to increase the strong coupling, due to the negative signs of the last two terms for tau and top couplings in the first curly bracket eq. (48) which dominate the bottom contribution. This is unlike equation (30), where the log's of Yukawa ratios had positive sign in front, (but were themselves negative). Here they are positive, but their sign in front is not positive for all bottom, tau, top couplings.

The second contribution comes from terms like $y_{\lambda}\left(\mu_{g}\right) / y_{\lambda}\left(M_{z}\right)$ and the third contribution comes from MSSM terms, $y_{\lambda}^{o}(0) / y_{\lambda}^{o}\left(M_{z}\right)$. These terms largely cancel because the running of Yukawa couplings below $\mu_{g}$ scale is the same in MSSM and EMSSM and we have same electroweak values for $y_{\lambda}\left(M_{z}\right)$, $y_{\lambda}^{o}\left(M_{z}\right)$ (these are inputs of our analysis); We also know that Yukawa effects in MSSM, coming in as ratios $y_{\lambda}^{o}(0) / y_{\lambda}^{o}\left(M_{z}\right)$ are small and the scale $\mu_{g}$ being in general heavy"t, we conclude that the last two curly brackets of eq. (48) do not solve the problem of how to reduce the small discrepancy between the predicted value for the strong coupling and its experimental value.

To conclude, it is not easy to reduce the strong coupling and increase the unification scale significantly, and it seems that the observation we made after eq.(13) is true in general for models which consider only complete additional representations.

\footnotetext{
${ }^{20}$ Considering $y_{\lambda}(0)$ smaller than $y_{\lambda}\left(\mu_{g}\right)$ is not allowed in this model because a rapid flow to infrared fixed points was assumed here and hence generation independent Yukawa couplings are possible for $y_{\lambda}(Q) / y_{\lambda}(0) \leq 1$, with $Q \leq M_{g}$

${ }^{21}$ The extra states are not protected by any chiral symmetry and therefore their mass is heavy
} 


\section{The large $n$ limit.}

Our analysis has so far assumed that perturbation theory works well up to the unification scale, and that the presence of a given number of states does not affect its convergence. However, for a large number of states and a large unified coupling one should carefully consider the limits of the two-loop perturbative expansion we applied in this work as well as in ref. [1]. It is the purpose of this section to explore the phenomenological implications for the case when these limits are reached, in the absence of Yukawa effects.

In the case of large $n$ and large $\alpha_{g}$ the perturbative expansion breaks down. An estimate of where breakdown occurs can be obtained by comparing two-loop beta function terms with three loop terms 2 or, equivalently, one-loop terms in the expansion of the anomalous dimensions of the fields with two-loop terms. Although the higher order terms have an additional power of coupling they are also proportional to $n$ and in the large $\mathrm{n}$ limit this will compensate for the additional coupling. This happens for $n \alpha \approx n^{2} \alpha^{2} /(4 \pi)$, or $n \alpha \approx \mathcal{O}(4 \pi)$. In the case of large $n$ the perturbation series in $\alpha$ as well as the perturbation series for anomalous dimensions of chiral fields can be resummed 15] to leading order $O(1 / n)$. This calculation was done by Jones in [15] and the reader is referred to this work for full details. We will use their results for the resummed anomalous dimensions of the light fields of the spectrum for the case the Yukawa couplings are ignored.

The anomalous dimension for any matter field is given by

$$
\gamma_{F}=-\sum_{k=1}^{3} \frac{\alpha_{k}}{2 \pi} G\left(\frac{\alpha_{k} n}{4 \pi}\right) C_{k}\left(R_{F}\right)
$$

Here $F$ stands for a matter field in the representation $R_{F}$ of the gauge group $S U(k){ }^{23}$. The function $\mathrm{G}$ is defined by

$$
G(\epsilon)=\frac{1}{2} \frac{\Gamma(3-2 \epsilon)}{\Gamma(2-\epsilon)^{2}} \frac{\sin (\pi \epsilon)}{\pi \epsilon}
$$

and has a pole for $\epsilon=3 / 2$ which sets the radius of convergence of the resummed series. For $G=1$ we recover the results of eq.(16). The running of the gauge couplings is now given by

$$
\begin{gathered}
\alpha_{i}^{-1}\left(M_{z}\right)=-\delta_{i}+\alpha_{g}^{-1}+\frac{b_{i}}{2 \pi} \ln \left[\frac{M_{g}}{M_{z}}\right]+\frac{n}{2 \pi} \ln \left[\frac{M_{g}}{\mu_{g}}\right]+\frac{1}{4 \pi} \sum_{j=1}^{3} \frac{n}{b_{j}^{\prime}}\left[2 \delta_{i j} \lambda_{j}-\frac{b_{i j}}{b_{j}}\right] \ln \left[\frac{\alpha_{g}}{\alpha_{j}\left(\mu_{g}\right)}\right] \\
+\frac{1}{4 \pi} \sum_{j=1}^{3} \frac{b_{i j}}{b_{j}} \ln \left[\frac{\alpha_{g}}{\alpha_{j}\left(M_{z}\right)}\right]+\mathcal{K}_{i}
\end{gathered}
$$

where $b_{i j}$ and $b_{j}$ are just the two-loop and one-loop beta functions for the MSSM and where $\lambda_{1}=0$, $\lambda_{2}=2, \lambda_{3}=3 . \mathcal{K}_{i}$ are the resummed corrections induced in the large $n$ limit ignoring Yukawa effects and are given by

$$
\mathcal{K}_{i}=\frac{1}{4 \pi} \sum_{j=1}^{3}\left(b_{i j}-2 \lambda_{j} \delta_{i j} b_{j}\right) \frac{1}{b_{j}^{\prime}} \int_{\alpha_{j}\left(\mu_{g}\right)}^{\alpha_{g}} \frac{d \alpha_{j}}{\alpha_{j}}\left[-1+G\left(\frac{\alpha_{j} n}{4 \pi}\right)\right]
$$

The effect of $\mathcal{K}_{i}$ 's on the unification scale and the strong coupling may now be readily computed to give

$$
\frac{M_{g}}{M_{g}^{o}}=e^{\frac{5 \pi}{14}\left(-\mathcal{K}_{1}+\mathcal{K}_{2}\right)}\left[\frac{\alpha_{g}}{\alpha_{g}^{o}}\right]^{31 / 21}\left[\frac{\alpha_{3}\left(M_{z}\right)}{\alpha_{3}\left(M_{z}\right)^{o}}\right]^{4 / 21}\left[\frac{\alpha_{g}}{\alpha_{1}\left(\mu_{g}\right)}\right]^{n /\left(12 b_{1}^{\prime}\right)}\left[\frac{\alpha_{g}}{\alpha_{2}\left(\mu_{g}\right)}\right]^{-39 n /\left(28 b_{2}^{\prime}\right)}\left[\frac{\alpha_{g}}{\alpha_{3}\left(\mu_{g}\right)}\right]^{4 n /\left(21 b_{3}^{\prime}\right)}
$$

\footnotetext{
${ }^{22}$ For this, expand the denominator of eq. (1) to get three loop terms

${ }^{23}$ For the $\mathrm{U}(1)$ factor $C_{k}\left(R_{F}\right)$ is $\frac{3}{5} Y^{2}$ where $Y$ is the usual weak hypercharge 15 .
} 


\begin{tabular}{|c|c|c|l|}
\hline$n$ & $\alpha_{g}$ & $M_{g} / M_{g}^{o}$ & $\alpha_{3}\left(M_{z}\right)$ \\
\hline \hline 30 & 0.1 & 1.359 & 0.12700 \\
\hline 30 & 0.2 & 1.760 & 0.12796 \\
\hline 30 & 0.3 & 2.043 & 0.12850 \\
\hline 40 & 0.1 & 1.355 & 0.12698 \\
\hline 40 & 0.2 & 1.745 & 0.12786 \\
\hline 40 & 0.3 & 2.019 & 0.12835 \\
\hline 50 & 0.1 & 1.353 & 0.12696 \\
\hline 50 & 0.2 & 1.737 & 0.12780 \\
\hline 50 & 0.3 & 2.006 & 0.12828 \\
\hline 60 & 0.1 & 1.351 & 0.12695 \\
\hline 60 & 0.2 & 1.732 & 0.12777 \\
\hline 60 & 0.3 & 1.999 & 0.12823 \\
\hline
\end{tabular}

Table 1: The values of $\alpha_{g}$, strong coupling at $\alpha_{3}\left(M_{Z}\right)$, and $M_{g} / M_{g}^{o}$ as a function of (large) n. We always have that $\left(n \alpha_{g}\right) /(4 \pi)<3 / 2$.

and

$$
\alpha_{3}^{-1}\left(M_{z}\right)=\alpha_{3}^{o-1}\left(M_{z}\right)-\frac{470}{77 \pi} \ln \left[\frac{\alpha_{g}}{\alpha_{g}^{o}}\right]+\sum_{j=1}^{3} \ln \left[\frac{\alpha_{g}}{\alpha_{j}\left(\mu_{g}\right)}\right]^{\omega_{j}}-\frac{17}{14 \pi} \ln \left[\frac{\alpha_{3}\left(M_{z}\right)}{\alpha_{3}^{o}\left(M_{z}\right)}\right]+\left(\mathcal{K}_{3}-\frac{12}{7} \mathcal{K}_{2}+\frac{5}{7} \mathcal{K}_{1}\right)
$$

with $\omega_{j}$ given in eq.(23). To use these equations it is necessary to compute the values of $\alpha_{j}\left(\mu_{g}\right)$. We do this by writing the equations equivalent to eq.(52) for $\alpha_{i}^{-1}\left(\mu_{g}\right)$ and eliminating the term $n /(2 \pi) \ln \left(M_{g} / \mu_{g}\right)$ between these equations for $\alpha_{i}$. This gives

$$
\alpha_{i}^{-1}\left(\mu_{g}\right)=\alpha_{g}^{-1}+\frac{5}{28} \frac{b_{i}^{\prime}}{n}\left(b_{2} \mathcal{K}_{1}-b_{1} \mathcal{K}_{2}\right)+\mathcal{K}_{i}-\frac{b_{i}^{\prime}}{n}\left[\frac{1}{\alpha_{g}}-\frac{1}{\alpha_{g}^{o}}\right]-\frac{2336}{231 n} \frac{b_{i}^{\prime}}{2 \pi} \ln \left[\frac{\alpha_{g}}{\alpha_{g}^{o}}\right]+\sum_{j=1}^{3} \ln \left[\frac{\alpha_{g}}{\alpha_{j}\left(\mu_{g}\right)}\right]^{v_{i j}}
$$

with

$$
v_{i j}=\frac{1}{4 \pi b_{j}^{\prime}}\left(b_{i j}+2 n \lambda_{j} \delta_{i j}\right)+\frac{b_{i}^{\prime}}{2 \pi}\left\{\frac{7}{132 b_{1}^{\prime}}, \frac{333}{28 b_{2}^{\prime}}, \frac{-88}{21 b_{3}^{\prime}}\right\}_{j}
$$

Note that the quantities $\mathcal{K}_{i}$ depend on $\alpha_{j}\left(\mu_{g}\right)$ as seen from eq.(53). For given $\alpha_{g}$ and large $n$ we solve (56) to get $\alpha_{j}\left(\mu_{g}\right)$ which are then used to compute the ratio $M_{g} / M_{g}^{o}$ and $\alpha_{3}\left(M_{z}\right)$ of eqs. (54), (55). The results for values of $n$ and $\alpha_{g}$ which avoid the pole in $G$ are presented in Table 1.

We see that the effects of a large number of states is very small and the results for $M_{g} / M_{g}^{o}$ and $\alpha_{3}\left(M_{z}\right)$ are in general insensitive to the variation of (large) $n$ and stay close to the MSSM values.

\section{The case of strong coupling.}

Our analysis to date has assumed that the couplings (even at large $\mathrm{n}$ ) remain in the perturbative domain between electroweak scale and unification scale. However, non-perturbative physics could be important below unification scale and in this section we will consider this possibility. In this case one apparently loses all the predictive power because the full beta functions are known only perturbatively and the perturbation series does not work beyond the scale of non-perturbative physics. In [8] it was argued 
that this is not the case because the ratios of the gauge couplings are driven towards stable infra-red fixed points. Since the couplings are initially large, the rate of flow to the infrared fixed point should be rapid. As a result, the low-energy values of these ratios are insensitive to their initial values and to the non-perturbative effects (provided the couplings initially lie in the domain of attraction of the fixed points), giving a reliable prediction for $\alpha_{3}\left(M_{z}\right)$ even in this case. Using the fixed points (FP) of the one-loop RGE to determine the boundary conditions of the gauge couplings at the decoupling scale of the additional massive states, it was found that a low value of $\alpha_{3}\left(M_{z}\right)$ is predicted, very close to the mean experimental value and in much better agreement than the perturbative MSSM result.

Here we wish to consider corrections to the above "fixed point" result in order to estimate the precision of the result and to establish whether the apparent improvement over the perturbative case is significant. To do this we shall continue to use the perturbative solution, but only in the domain where it is applicable. As we shall see in this region one may see the RG flow to the fixed points but there are calculable corrections. The first point to make is that even using the improved perturbation sum discussed in the last section one requires $n \alpha \leq \mathcal{O}(6 \pi)$. We can only calculate those effects below the non-perturbative domain at the scale $M_{o}$ where the gauge couplings enter the perturbative domain and as we have just remarked this starts at quite small values of $\alpha$. Thus our error estimates will necessarily be somewhat rough. There are two corrections to the "fixed point" calculation, deriving from the relations among the gauge couplings at the decoupling scale $\mu_{g}$, and from the uncertainty in their exact values as they leave the non-perturbative region to enter the perturbative domain. Here we analyse them briefly.

The first correction arises because, even if one sticks to the one-loop beta functions, due to the finite energy range involved the ratios of couplings are not driven quite to the fixed point. Therefore the boundary condition we used for the evolution of the couplings below $\mu_{g}$ is not the "fixed-point" ratio [8]

$$
R_{j i}\left(\mu_{g}\right) \equiv \frac{\alpha_{j}\left(\mu_{g}\right)}{\alpha_{i}\left(\mu_{g}\right)}=R_{j i}^{*}
$$

with $R_{j i}^{*}=b_{i}^{\prime} / b_{j}^{\prime}$. Instead, the boundary condition for the running of the gauge couplings below the decoupling scale ${ }^{26}$ is a "quasi-fixed-point" (QFP) relation for the ratio of the gauge couplings. This relation exactly takes into account a one-loop running for the gauge couplings above $\mu_{g}$ scale (just as in FP case) and the finite range of energy. This QFP relation is

$$
R_{j i}\left(\mu_{g}\right) \equiv \frac{\alpha_{j}\left(\mu_{g}\right)}{\alpha_{i}\left(\mu_{g}\right)}=\frac{R_{j i}^{*}}{1-\left[1-\frac{R_{j i}^{*}}{R_{j i}\left(M_{o}\right)}\right] \frac{\alpha_{i}\left(\mu_{g}\right)}{\alpha_{i}\left(M_{o}\right)}}
$$

This can be easily deduced by integrating the one-loop differential eqs for the gauge couplings above $\mu_{g}$. Using this QFP boundary condition for the gauge couplings evolution below $\mu_{g}$ scale, one gets a value for $\alpha_{3}\left(M_{z}\right)$ which is typically smaller than that of FP case. The results depend on the value of $R_{i j}\left(M_{o}\right)$, which enters (59), but this dependence is relatively weak and the prediction for $\alpha_{3}\left(M_{z}\right)$ stays below MSSM value. This is somewhat expected, as we know that two-loop corrections in MSSM increase $\alpha_{3}\left(M_{z}\right)$ from its rather good one loop prediction 27 , taking it away from the current experimental upper limit. As eq. (59) ignores the two loop evolution' effects between $\mu_{g}$ and $M_{o}$, we get a smaller $\alpha_{3}\left(M_{z}\right)$ than in MSSM.

\footnotetext{
${ }^{24}$ We will however ask that $n \alpha \leq \mathcal{O}(4 \pi)$ instead, because we will not use the resummed perturbation series, and this relation marks the limit where it becomes important.

${ }^{25}$ In two-loop running of gauge couplings the effective decoupling scale is $\mu_{g}$, the bare mass of the heavy spectrum

${ }^{26}$ Below this scale a MSSM like spectrum applies

${ }^{27}$ In MSSM a one-loop calculation gives $\alpha_{3}\left(M_{z}\right) \approx 0.117$ while two-loop calculation increases it to $\approx 0.126$
} 
This brings us to the second correction which consists of two-loop or higher order (gauge and Yukawa) effects between $\mu_{g}$ and $M_{o}$. This affects the relation (59) and therefore the low energy predictions for the strong coupling. We will consider here only the gauge effects. Their effect is to change the expression of $R_{j i}\left(\mu_{g}\right)$ such as to increase the value of the strong coupling at electroweak scale. The increase overcomes the QFP decrease in $\alpha_{3}\left(M_{z}\right)$, bringing it closer to MSSM prediction. This conclusion is subject to the corrections due to the unknown values of the gauge couplings at $M_{o}$ (or equivalently $Z\left(M_{o}\right)$ ), where they enter the perturbative domain as we lower the scale, but there is a systematic effect increasing the value of $\alpha_{3}\left(M_{z}\right)$ even for sizeable changes in the boundary conditions.

Our analysis is limited because the range between $\mu_{g}$ and $M_{o}$ is quite small and the calculable corrections to the fixed point result correspondingly small. However this does not mean the full nonperturbative correction to the fixed point result is small. An estimate of these corrections coming from the higher order and quasi fixed point terms discussed above may be obtained by noting that there is a focussing effect of the fixed point following in the perturbative flow domain which reduces the contribution of such effects by the factor $\frac{\alpha_{i}\left(\mu_{g}\right)}{\alpha_{i}\left(M_{o}\right)}$ (cf eq(59)). Using this factor with $\alpha_{i}\left(M_{o}\right)$ limited by the perturbative condition $n \alpha \leq \mathcal{O}(4 \pi)$ together with the assumption of $O(1)$ deviations from the QFP boundary condition we arrive at a reasonably conservative estimate for these errors of $\mathcal{O}( \pm 0.01)$ for $n=12$, increasing for larger values of $n$.

\section{Summary and Conclusions}

For the case of perturbative evolution of the gauge and Yukawa couplings up to the unification scale, we derived an analytical method to determine the two-loop unification predictions for the value of unification scale, the intermediate mass scale and the value of the strong coupling at $M_{z}$ in models with the MSSM spectrum augmented by additional massive representations filling out complete $S U(5)$ representations. The effects of the two-loop terms involving Yukawa couplings are in general relatively small and model-dependent. For the models we considered, $\alpha_{3}\left(M_{z}\right)$ cannot be lowered below the MSSM value, even with Yukawa effects present, keeping $\alpha_{3}\left(M_{z}\right)$ above the mean experimental value. However the sign of the effect is not universal, so it is possible a richer structure in Yukawa sector could reduce the strong coupling. We also showed that the unification scale is not changed significantly by the top, bottom and tau Yukawa coupling effects. Using the large $\mathrm{n}$ resummed perturbation series we showed that the results are stable in the limit of inclusion of a large number of complete $S U(5)$ representations and stay close to MSSM predictions. Finally we considered the case that unification occurs at strong coupling with perturbative analysis breaking down below the unification scale. Because of the fixed point structure in the RGEs (at one-loop level) one may still make a prediction for $\alpha_{3}\left(M_{z}\right)$. However threshold and two loop effects above the decoupling scale for the heavy states can be sizeable. As a result the low estimate for $\alpha_{3}\left(M_{z}\right)$ obtained using fixed point boundary conditions at the decoupling scale is increased for large $\mathrm{n}$ towards the MSSM value. Overall we estimate an irreducible error in the strong coupling determination of $\alpha_{3}\left(M_{z}\right)$ of $O(0.01)$ coming from the residual sensitivity to the initial values of the ratios of the couplings as they enter the perturbative domain and from two-loop corrections to $R_{j i}\left(\mu_{g}\right)$ above $\mu_{g}$.

\section{Acknowledgments}

D.G. gratefully acknowledges the financial support from the part of University of Oxford and Oriel College (University of Oxford). G. A.-C. acknowledges the financial support for his work on this project from P.P.A.R.C., the Foundation Blanceflor Boncompagni-Ludovisi and the Swiss National Science Foundation. 


\section{Appendix}

We have the following expressions for $T\left(R_{\sigma}\right)$ for the fundamental representation

$$
\delta^{a b} T(R)=\operatorname{Tr}\left(T^{a} T^{b}\right)=\frac{1}{2} \delta^{a b}
$$

For a given flavour the value of $T\left(R_{\sigma}\right)$ is $T\left(R_{\sigma}\right)=1 / 2 .\left(T\left(R_{\sigma}\right)=1\right.$ for conjugate pairs of fields).

For the adjoint representation we have

$$
\left(T^{a}\right)_{b c}=-i f^{a b c}
$$

and therefore, for $S U(N)$ group

$$
\delta^{a b} T(G)=f^{a c d} f^{b c d}=N \delta^{a b}
$$

where the structure constants $f^{a b c}$ are given by

$$
\left[T^{a}, T^{b}\right]=i f^{a b c} T^{c}
$$

The values of $\mathcal{A}$ used in text (20) in the context of a MSSM like superpotential and considering only the Yukawa couplings for top, bottom and tau is given by (see for example [16])

$$
\mathcal{A}_{\nu}(F)=\left(\begin{array}{rrrrrrrr} 
& l_{L_{3}} & q_{L_{3}} & e_{R_{3}} & u_{R_{3}} & d_{R_{3}} & H_{1} & H_{2} \\
\nu=\tau: & -1 & 0 & -2 & 0 & 0 & -1 & 0 \\
\nu=b: & 0 & -1 & 0 & 0 & -2 & -3 & 0 \\
\nu=t: & 0 & -1 & 0 & -2 & 0 & 0 & -3
\end{array}\right)
$$

The running of Yukawa couplings in one-loop, for a MSSM like superpotential can be rewritten, using [16], as follows (we ignore the first two generations' contribution)

$$
\left(\begin{array}{c}
y_{\tau}(t) \\
y_{b}(t) \\
y_{t}(t)
\end{array}\right)=\left(\begin{array}{ccc}
\frac{35}{122} & \frac{-9}{61} & \frac{3}{122} \\
\frac{-3}{61} & \frac{12}{61} & \frac{-2}{61} \\
\frac{1}{122} & \frac{-2}{61} & \frac{21}{122}
\end{array}\right)\left(\begin{array}{c}
\frac{d \ln y_{\tau}}{d t} \\
\frac{d \ln y_{b}}{d t} \\
\frac{d \ln y_{t}}{d t}
\end{array}\right)+\left(\begin{array}{ccc}
\frac{143}{305} & \frac{30}{61} & \frac{-40}{61} \\
\frac{-23}{915} & \frac{21}{61} & \frac{160}{183} \\
\frac{136}{915} & \frac{27}{61} & \frac{136}{183}
\end{array}\right)\left(\begin{array}{c}
\alpha_{1}(t) \\
\alpha_{2}(t) \\
\alpha_{3}(t)
\end{array}\right)+\mathcal{O}\left(\alpha^{2}\right)
$$

For a family symmetric superpotential we used in text (41)

$$
W=\sum_{i, j=1}^{3}\left(h_{i j}^{u} Q_{i} U_{j} H_{2}^{i j}+h_{i j}^{d} Q_{i} U_{j} H_{1}^{i j}+h_{i j}^{l} L_{i} e_{j} H_{1}^{i j}\right)
$$

we have that

$$
\mathcal{B}_{\nu}(F)=\left(\begin{array}{rrrrrrrr} 
& l_{L_{j}} & q_{L_{j}} & e_{R_{j}} & u_{R_{j}} & d_{R_{j}} & H_{1} & H_{2} \\
\nu=\tau: & -3 & 0 & -6 & 0 & 0 & -1 & 0 \\
\nu=b: & 0 & -3 & 0 & 0 & -6 & -3 & 0 \\
\nu=t: & 0 & -3 & 0 & -6 & 0 & 0 & -3
\end{array}\right)
$$

For the above class of superpotential, the Yukawa couplings running in one loop can be written as follows

$$
\left(\begin{array}{c}
y_{\tau}(t) \\
y_{b}(t) \\
y_{t}(t)
\end{array}\right)=\left(\begin{array}{ccc}
\frac{15}{146} & \frac{-2}{73} & \frac{1}{146} \\
\frac{-2}{219} & \frac{20}{219} & \frac{-5}{219} \\
\frac{1}{438} & \frac{-5}{219} & \frac{13}{146}
\end{array}\right)\left(\begin{array}{c}
\frac{d \ln y_{\tau}}{d t} \\
\frac{d \ln y_{b}}{d t} \\
\frac{d \ln y_{t}}{d t}
\end{array}\right)+\left(\begin{array}{ccc}
\frac{13}{73} & \frac{18}{73} & \frac{-8}{73} \\
\frac{7}{1095} & \frac{13}{73} & \frac{80}{219} \\
\frac{232}{3285} & \frac{15}{73} & \frac{232}{657}
\end{array}\right)\left(\begin{array}{c}
\alpha_{1}(t) \\
\alpha_{2}(t) \\
\alpha_{3}(t)
\end{array}\right)+\mathcal{O}\left(\alpha^{2}\right)
$$




\section{References}

[1] D. Ghilencea, M. Lanzagorta, G. G. Ross, Nucl. Phys. B 511 (1998) 3-24.

[2] W.Pokorski, G.G.Ross, preprint OUTP-97-34-P(1997), hep-ph/9707402 and references therein.

[3] K.R. Dienes, A.E. Faraggi and J. March-Russell, Nucl. Phys. B 467 (1996) 44.

[4] Review of Particle Data, Phys. Rev. D 54 (1996), 83.

[5] P. Ginsparg, Phys. Lett. B 197 (1987), 139;

V.S. Kaplunovsky, Nucl. Phys. B 307 (1988), 145;

Erratum: ibid. B 382 (1992), 436.

[6] K.R. Dienes, Phys. Rept. 287 (1997) 447 and references therein.

[7] Luis Ibanez and Graham G. Ross, Phys. Lett. B 332 (1994) 100;

Graham G. Ross, Phys. Lett. B 364 (1995) 216.

[8] D. Ghilencea, M. Lanzagorta, G. Ross, Phys. Lett. B 415 (1997), 252.

[9] V.A. Novikov et al, Phys. Lett. B 166 (1986), 329.

V.A. Novikov et al, Nucl. Phys. B 277 (1986), 456.

[10] N. Arkani-Hamed and H. Murayama, preprint LBNL-40460, hep-th/9707133

[11] M. Shifman, Int.J.Mod.Phys. A11 (1996) 5761, Talk at the International Workshop on Supersymmetry and Unification of Fundamental Interactions SUSY-96, University of Maryland, College Park, MD 20742, USA.

Other related works:

M.A. Shifman, A.I. Vainshtein, Nucl.Phys. B 277 (1986), 456.

M.A. Shifman, A. I. Vainshtein, Nucl. Phys. B 359 (1991), 571.

H. S. Li, K. T. Mahanthappa, Phys. Lett. B 319 (1993), 152.

M. Dine, Y. Shirman, Phys. Rev. D 50 (1994), 5389.

M. Shifman, preprint TPI-MINN-97/09-T, UMN-TH-1535-97, hep- ph/9704114

J. Hisano, M. Shifman, preprint TPI-MINN-97/14-T, UMN-TH-1543-97, hep-ph/9705417.

[12] N.Arkani-Hamed, H. Murayama, preprint LBNL-40346, hep-ph/9705189

[13] I. Jack, D.R.T. Jones and C.G. North, Phys.Lett. B 386 (1996) 138.

[14] W. de Boer et al., preprint IEKP-KA/96-04, hep-ph/9603350

[15] P.M. Ferreira, I. Jack, D.R.T. Jones and G.G. North, hep-ph/9705328

[16] J.E. Bjorkman and D.R.T. Jones, Nucl. Phys. B 259 (1985) 533. 